\title{
Extrinsic transient diffusion in silicon
}

\author{
Martin D. Giles \\ University of Michigan, Department of Electrical Engineering and Computer Science, Ann Arbor, \\ Michigan 48109-2122
}

(Received 21 January 1991; accepted for publication 27 February 1991)

\begin{abstract}
The effect of extrinsic background doping on the transient enhancement of dopant diffusion for an ion-implanted dopant is investigated to gain insight into the role of point defect charge states. The transient effect is found to be greatly increased for extrinsic background doping of the same type as the implanted ion, and reduced for background doping of the opposite type. Analysis of the relative enhancements for boron and phosphorous allows the position of the donor and acceptor levels for the silicon self-interstitial to be extracted. The results are in good agreement with earlier work based on extrinsic oxidation-enhanced diffusion.
\end{abstract}

In recent years there has been considerable work aimed at improving our understanding of the mechanisms of dopant diffusion in silicon. A number of workers ${ }^{1-6}$ have developed related formalisms describing the coupling of point defects with dopant to account for the range of effects seen experimentally ${ }^{7}$ such as concentration-dependent diffusion, oxidation-enhanced diffusion, the emitter push effect, transient damage enhanced diffusion, and many others. $\mathrm{Nu}$ merical simulation using these models requires knowledge about the properties of point defects, dopants, and their interaction. Although the silicon self-interstitial is believed to play a crucial role for many dopants, direct observation and measurement of its properties has been surprisingly difficult. Most experimental information has been inferred from measurement of the effect of interstitials on dopant diffusion or extended defect growth.

One crucial parameter for simulation of extrinsic silicon is the charge state properties of point defects. These have been measured at low temperatures for vacancies ${ }^{8}$ and also proposed for interstitials, ${ }^{9}$ although the interstitial values are not as generally accepted. For defects with charge states within the band gap, the equilibrium concentration of the charged states is a function of the local Fermi level. Local variations in doping lead to variations in point defect concentration which in turn lead to variations in dopant diffusivity. Knowledge of these effects is essential to accurate simulation of point defect coupled dopant diffusion under extrinsic doping conditions. In this letter we will describe experiments which allow the charge state energy levels of a silicon self-interstitial to be extracted from variations in transient damage enhanced diffusivity of an ionimplanted dopant with background doping level. This approach is similar to earlier work using oxidation-enhanced diffusion, ${ }^{10}$ but the nature of transient diffusion allows a clearer evaluation of the results.

We have previously presented a simple, quantitative physical model for transient diffusion of ion-implanted dopants in silicon below the amorphization threshold. ${ }^{11}$ For the purpose of this study, ion implantation provides a large, fixed injection of a known quantity of interstitials which enhance dopant diffusion. The enhancement persists until the excess dissipates through recombination or diffusion into the bulk. The initial excess is determined by the ion implantation conditions and is not sensitive to the level of background doping in the silicon wafer. This compares favorably with interstitital injection by oxidation since that injection is much smaller and the oxidation rate varies somewhat with substrate doping.

One effect of substrate background doping is to alter the distribution of charge states of any point defects that are present. Since the contribution of a point defect.to dopant diffusion depends on its charge state, there is a corresponding variation in dopant diffusivity. The wellknown concentration dependent diffusivity of dopants arises directly from this effect. In the case of transient enhanced dopant diffusion, the magnitude of the transient varies with background doping for the same initial damage distribution. This letter presents analysis of the variation as a function of background doping which allows the charge states of interstitials to be estimated, following a similar approach to an earlier analysis of extrinsic oxidation enhanced diffusion. ${ }^{10}$

These experiments used $\langle 100\rangle 7 \mu \mathrm{m} p$-on- $p^{+}$epitaxial wafers. A $125 \AA$ A screen oxide was grown by dry oxidation and the wafers implanted either with $5 \times 10^{15} \mathrm{~cm}^{-2}$ boron-11 at $50 \mathrm{keV}, 5 \times 10^{15} \mathrm{~cm}^{-2}$ arsenic at $100 \mathrm{keV}$ or left unimplanted. The wafers were then capped with $2000 \AA$ of deposited oxide and annealed at $1150{ }^{\circ} \mathrm{C}$ for $4 \mathrm{~h}$ to create a uniform background doping over the first micron of the substrate. The doping levels after annealing were $5 \times 10^{20}$ $\mathrm{cm}^{-3}$ for boron- 11 and $8 \times 10^{20} \mathrm{~cm}^{-3}$ for arsenic. The oxide cap was stripped and $125 \AA$ oxide grown by dry oxidation before implanting with boron-10 at $70 \mathrm{keV}$ and a dose of $10^{14} \mathrm{~cm}^{-2}$. This dose was chosen to be below threshold for the formation of extended defects during annealing, and well below the amorphization threshold for the lattice. The wafers were then annealed at $900^{\circ} \mathrm{C}$ for 30 min. Similar experiments were performed to examine phosphorus transient diffusion using background boron- 11 and arsenic doses of $2 \times 10^{16} \mathrm{~cm}^{-2}$, a final phosphorus implant at $150 \mathrm{keV}$ and a dose $7 \times 10^{13} \mathrm{~cm}^{-2}$, and annealing at $800^{\circ} \mathrm{C}$ for $75 \mathrm{~min}$. Samples were analyzed by secondaryion mass spectrometry (SIMS) using a CAMECA IMS- $3 f$ system.

Simulation of the diffusion of dopant and point defects was performed using a modified version of the SUPREM IV 
computer program ${ }^{3,12}$ including full coupling between dopant and defects, assuming that dopant-defect pairs remain in local equilibrium, and assuming equilibrium between the charge state within each species. We assume that only a small fraction of the point defects are associated with dopant at any one time, so that the concentration of a particular pair is proportional to the concentration of the corresponding free point defect. The interstitial diffusivily was described by $D_{I} C F=360 \exp (-4.8 / k T) \mathrm{cm}^{2} / \mathrm{s}$ based on earlier work ${ }^{11}$ and the vacancy diffusivity by the default value of $D_{V} C v=0.4 \exp (-4.0 / k T) \mathrm{cm}^{2} / \mathrm{s}$, since this was not critical to the calculated results. The fractional interstitial component of diffusion for boron and phosphorus was assumed to be 0.95 and 1.00 , respectively. The two adjustable parameters available to fit the experimental results were the position of the silicon self-interstitial donor and acceptor energy levels within the silicon band gap. The largest diffusion occurs when the background doping is of the same type as the second, low-dose implant, so this gives the most sensitive measure of the diffusion enhancernent. Boron diffusion allows the donor energy level to be fitted; phosphorus diffusion allows the acceptor energy level to be fitted. Simulation used the measured background doping levels to determine the Fermi level and fields for the transient diffusion calculation. We included the normal diffusion that would be expected for these times, temperaturcs, and doping levels, although the values are small compared to the observed effects. For boron and phosphorous, the equilibrium values of $\sqrt{D t}$ at these temperatures is 100 and $34 \AA$ A, respectively.

In the dilute approximation, the ratio of charged to uncharged point defects is given by relation such as ${ }^{7}$

$$
\alpha=\frac{C_{I^{+}}}{C_{I^{0}}}=\frac{\theta_{I^{+}}}{\theta_{l^{0}}} \exp \left(\frac{E_{I^{+}}-E_{f}}{k T}\right) .
$$

where $C_{I^{+}}$is the concentration of positively charged interstitials, $\theta_{I}+$ is the number of degrees of freedom, $E_{I}+$ is the donor energy level, and $E_{f}$ is the Fermi level. Fitting simulation to experiment determines the value of the quantity $\alpha$. Energy levels are inferred from $\alpha$ assuming $\theta_{I^{+}}=\theta_{l^{0}}$ $=\theta_{I^{-}}$.

Figure 1 shows simulation and experiment for annealing of boron under three background doping conditions, together with fitted simulation results. The experimental results show the expected variation in transient with background doping, being suppressed in $n$-type silicon and enhanced in p-type silicon. Comparing experiment to simulation, the intrinsic doping experimental data shows somewhat more diffusion at the surface and less around the peak of the implant than predicted based on the point defect coefficients given above. These differences were not seen for samples with background doping or with phosphorus diffusion profiles, and are still under investigation. The position of the donor interstitial energy level was adjusted to give the best fit to the enhanced transient profile with boron background doping, where a value of $0.39 \mathrm{eV}$ above the valence band gave the best agreement. Figure 2

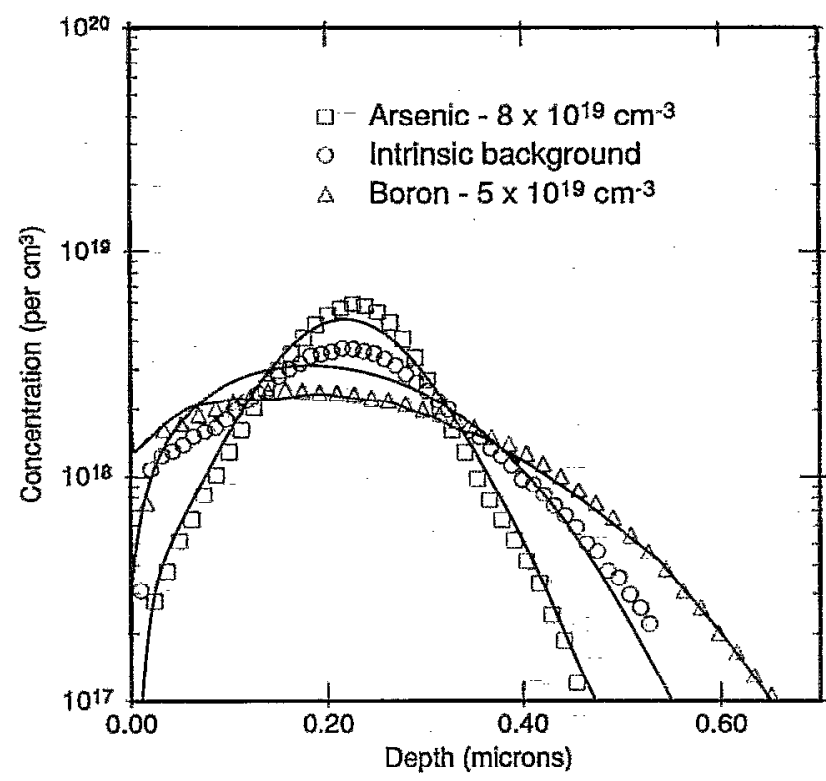

FIG. 1. Transient enhanced diffusion of boron implanted at $70 \mathrm{keV}$ and a dose of $10^{14} \mathrm{~cm}^{-2}$ and annealed at $900^{\circ} \mathrm{C}$ for $30 \mathrm{~min}$, as a function of uniform background doping level. The solid lines are the fitted simulation results.

shows the variation in calculated transient profile with fitting parameter, indicating a fitting accuracy of around 50 meV.

Figure 3 shows simulation and experiment for annealing of phosphorus under similar background doping conditions, again showing the variation in transient diffusivity. This dopant allows the acceptor interstitial energy to be fitted, resulting in a value of $0.09 \mathrm{eV}$ below the conduction band. Using the fitted values, simulations for all three background doping conditions_show good agreement with

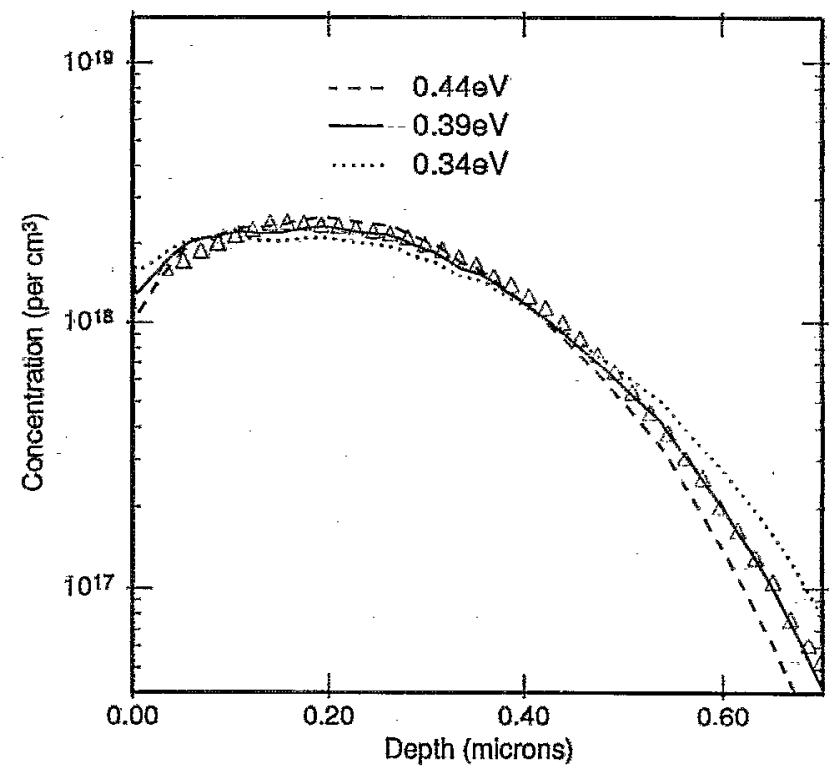

FIG. 2. Yariation in simulation result with the position of the donor interstitial energy level for transient enhanced diffusion of boron with uniform boron-background concentration of $5 \times 10^{19} \mathrm{~cm}^{-3}$. 


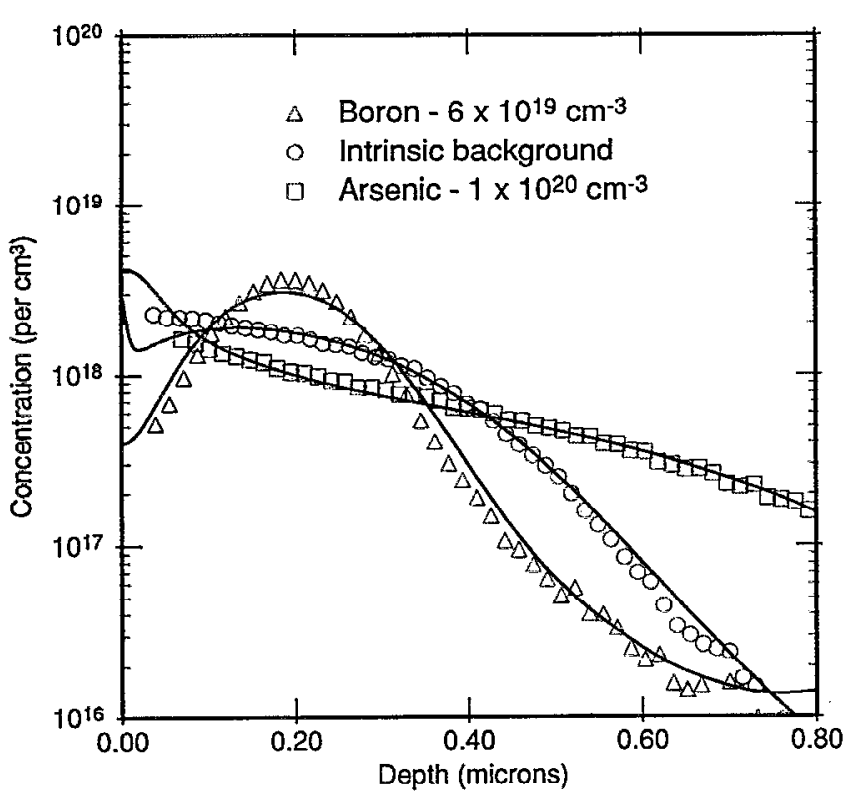

FIG. 3. Transient enhanced diffusion of phosphorus implanted at $150 \mathrm{keV}$ and a dose of $7 \times 10^{13} \mathrm{~cm}^{-2}$ and annealed at $800^{\circ} \mathrm{C}$ for $75 \mathrm{~min}$, as a function of uniform background doping level. The solid lines are the fitted simulation results.

the experimental results. The values obtained here from analysis of the transient enhanced diffusion effect are the same as those obtained previously from analysis of the variation of oxidation-enhanced diffusion with background doping. ${ }^{10}$ The fitted value for the donor level is very close to the value of $E_{V}+0.4 \mathrm{eV}$ previously proposed ${ }^{9}$ but the acceptor level differs significantly from the proposed value of $E_{C}-0.4 \mathrm{eV}$. Since we wish to use these coefficients in models of diffusion at high temperatures, and the possibility of a change in defect structure between low- and hightemperature measurements cannot be excluded, it seems reasonable to prefer coefficients fitted to high-temperature experimental data for use in simulating point defect coupled diffusion.

The experimental data presented here was obtained while the author was with AT\&T Bell Laboratories, Murray Hill, NJ.

${ }^{1}$ D. Mathiot and J. C. Pfister J. Appl. Phys. 55, 3518 (1984).

${ }^{2}$ B. J. Mulvaney and W. B. Richardson, Appl. Phys. Lett. 51, 1439 (1987).

${ }^{3}$ M. E. Law, PhD thesis, Stanford University, Stanford, CA 1988.

${ }^{4}$ M. Orlowski, Appl. Phys. Lett. 53, 1323 (1988).

${ }^{5}$ N. E. B. Cowern, J. Appl. Phys. 64, 4484 (1988).

${ }^{6}$ F. F. Morehead and R. F. Lever, J. Appl. Phys. 66, 5349 (1989).

${ }^{7}$ P. M. Fahey, P. B. Griffin, and J. D. Plummer, Rev. Mod. Phys. 61, 289 (1989).

${ }^{8}$ G. D. Watkins, Inst. Phys. Conf. Ser. 23, 1 (1975).

${ }^{9}$ W. Frank, Inst. Phys. Conf. Ser. 23, 23 (1975).

${ }^{10} \mathrm{M}$. D. Giles, IEEE Trans. CAD 8, 460 (1989).

${ }^{11}$ M. D. Giles, J. Electrochem. Soc. 138, 1160 (1991).

${ }^{12}$ M. E. Law, C. S. Rafferty, and R. W. Dutton, "New $n$-well fabrication techniques based on $2 \mathrm{~d}$ process simulation" IEDM Technical Digest (IEEE, New York, 1986), pp. 518-521. 